\title{
Evaluation of the inner choroid using OCT angiography
}

\author{
Federico Corvi $\mathbb{C}^{1,2,3} \cdot$ Li Su $^{1,2,4} \cdot$ Srinivas R. Sadda ${ }^{1,2}$
}

Received: 2 September 2020 / Revised: 16 September 2020 / Accepted: 29 September 2020 / Published online: 12 October 2020

(c) The Author(s), under exclusive licence to The Royal College of Ophthalmologists 2020

\begin{abstract}
The advent of optical coherence tomography angiography (OCTA) has allowed a qualitative and quantitative analysis of the retinal vasculature and the choriocapillaris. With the use of OCTA, several studies evaluated the changes in the choriocapillaris showing how this vascular structure plays a significant role in the pathogenesis of different conditions. This article reviews the current methods of analysis of the choriocapillaris and the relevant findings in different chorioretinal diseases.
\end{abstract}

\section{Introduction}

The choroid is a vascular structure that plays an essential role in the homeostasis of the retina. It provides oxygen and other nutrients to the outer layers of the retina and retinal pigment epithelium (RPE) and removes the metabolic waste products from the outer retina [1]. Thus, not surprisingly, dysfunction of the choroid is involved in the pathogenesis and progression of various different retinal diseases. For this reason, accurate and reproducible imaging of the choroid may be of value to understand disease pathophysiology.

Optical coherence tomography angiography (OCTA) is a relatively new imaging technology that offers the opportunity to image the retinal and inner choroidal circulation without the need for dye injection [2]. OCTA employs motion contrast to detect blood flow and acquires three-dimensional volumetric information of the retina and choroid to provide

These authors contributed equally: Federico Corvi, Li Su

Srinivas R. Sadda

ssadda@doheny.org

1 Doheny Image Reading Center, Doheny Eye Institute, Los Angeles, CA, USA

2 Department of Ophthalmology, David Geffen School of Medicine at UCLA, Los Angeles, CA, USA

3 Eye Clinic, Department of Biomedical and Clinical Science "Luigi Sacco", Sacco Hospital, University of Milan, Milan, Italy

4 Department of Ophthalmology, Shanghai General Hospital (Shanghai First People's Hospital), Shanghai Jiao Tong University School of medicine, Shanghai, China high-resolution, depth-resolved segmentation of the different vascular layers, including the thin inner choroid [2].

In this review we define the current role of OCTA in the imaging of the inner choroid. After a brief overview of the basic principles of this technique, we will describe the main observations regarding OCTA imaging of the inner choroid in various conditions.

\section{Anatomy of the choroid}

The choroid is the posterior portion of the uvea extending from the optic disc to the pars plana [3]. It is composed predominantly of vascular tissue and originates from the long and the short posterior ciliary arteries (PCAs), where the short PCAs perforate the sclera around the optic nerve and then provide perpendicular terminal arterioles forming the capillary lamina of choroid, and the long PCAs run anteriorly between the sclera and choroid forming anastomotic connections with the anterior choroid and joining the anterior ciliary arteries $[4,5]$.

Five different layers of the choroid may be discerned, from inner to outer: Bruch's membrane, choriocapillaris, Sattler's layer, Haller's layers, and the suprachoroidal space. The Bruch's membrane is the innermost layer formed by a connective tissue which supports the adhesion, migration, and differentiation of RPE cells and serves as a barrier between the retina and choroid.

The choriocapillaris is formed by a single continuous layer of wide-lumen fenestrated capillaries arranged in one thin sheet plane. The capillaries are arranged into a sequence of small fenestrated hexagonal-shaped lobules that become progressively larger from the center to the 
periphery [6]. In fact, the choriocapillaris is characterized by a high flow rate in the macular area with fewer intravascular space in order to provide oxygen, nutrients, and remove the metabolic waste products from the more densely packed RPE and photoreceptors [7].

Below the choriocapillaris is an intermediate layer of medium-caliber vessels, known as the Sattler layer, and a deeper layer of large-caliber vessels, known as the Haller layer. Lastly, the suprachoroidal space is a transitional zone between choroid and sclera containing collagen fibers, fibroblasts and melanocytes [3, 8].

\section{Optical coherence tomography angiography}

OCTA has revolutionized imaging in ophthalmology, offering the opportunity to noninvasively visualize different retinal capillary and inner choroidal layers $[9,10]$. In particular, this technology is based on the recognition of the intrinsic movement of the particles in the biological tissue to provide a reconstruction of the retinal and choroidal vessels. OCTA technology is based on the repetition of multiple OCT scans at the same location, followed by the detection of differences between these scans over time. Of note, the depiction of retinal and choroidal vessels is the result of blood flow detection and for this reason the vessel representation should be considered as the lumen rather than the entire vessel (lumen + vessel wall).

Several OCTA devices are available in clinical practice, and can be broadly divided into spectral domain (SD) and swept source (SS). This distinction is based on the light source of the device, $840 \mathrm{~nm}$ for the SD and $1050 \mathrm{~nm}$ for the SS, resulting in a different penetration of the signal through the RPE, pigment deposits, drusen, and other blocking structures, thereby producing a different visualization of the choroidal layers [11-14]. Furthermore, SDOCT devices are characterized by a broader bandwidth light source which is coupled with a spectrometer, while SS-OCT devices are equipped with photodetectors and a tuneable laser light source that operates through a range of frequencies. Although the speed of SD-OCT devices has progressively increased (with some devices operating at $100 \mathrm{KHz}$ ), SS-OCT devices are generally faster.

The main advantage of OCTA is the opportunity to perform detailed and high-resolution qualitative and quantitative analysis. The qualitative analysis is based on the recognition of specific features such as macular neovascularization (MNV) or areas of non-perfusion areas which may be of relevance to clinical practice $[14,15]$. The quantitative analyses may not be as relevant to current practice, but are invaluable in research studies, particularly in longitudinal assessments, and may give new insights into the pathogenesis of disease [2].
OCTA images combine the structural information of a standard OCT scan with blood flow visualization. The moving elements are commonly coded as bright/white pixels on the OCTA scans to represent blood flow. While both structural OCT and OCTA can and should be viewed on B-scans as well as en face images, OCTA is most commonly assessed using the en face representation. This may be because this view more closely parallels dye-based angiography. However, the most reliable way to view and interpret OCTA images is to evaluate both the en face OCTA image and the B-scan with flow overlay, as well as the corresponding en face structural OCT image.

\section{OCTA and choriocapillaris}

Different strategies have been adopted in order to provide a representation of the choriocapillaris. The typical single en face choriocapillaris image has a granular appearance of alternating white and black pixels, which makes it difficult to distinguish the capillary detail. This is thought to be due to the limited resolution of current OCTA technology, noise and as well as the dynamic aspect of the choriocapillaris circulation.

The anatomic position of the choriocapillaris is just below the Bruch's membrane and extending to the inner border of Sattler's layer. However, the imaging of the choriocapillaris with OCTA may not be as simple as positioning a slab in the proper anatomic location as there are several factors that may influence its representation. First, is the variability of the thickness of $\mathrm{CC}$, which is $10 \mu \mathrm{m}$ under the fovea and $7 \mu \mathrm{m}$ moving to the periphery albeit with some undulations/fluctuations [8]. Thus, the outer border of the choriocapillaris may not be located at a consistent offset from Bruch's membrane, which may introduce inaccuracy despite an otherwise "correct" anatomic segmentation. To make matters worse, many devices offset the inner border of the CC with respect to the centreline of the RPE band. As the RPE can also vary in thickness across the macula, this can lead to variability in the position of the inner boundary of the CC slab when a fixed offset is used. As a result of these subtle segmentation inaccuracies, many researchers have resorted to using a deeper slab positioned in the inner choroid $[2,15,16]$. The resultant OCTA en face image does seem to still resemble the pattern of the $\mathrm{CC}$, and many have speculated that what is being imaged is the projection artifact from the overlying CC. However, it must be acknowledged that this deeper slab image may be confounded by the inner choroidal structures that may be present at this location-a phenomenon which may be exacerbated when signal compensation approaches are applied. Therefore, we have proposed the term "inner choroidal slab" to refer to this deeper position, and have 
shown that these deeper slabs may yield more repeatable measurements, albeit at the expense of inaccuracy in the representation of the physical CC.

Thus, given these tradeoffs, there is no consensus on the best position and thickness of the CC slab. Byon et al. [16] suggested to adopt the processing strategy that yields the most repeatable results and for this reason, they investigated the impact of processing technique and slab selection on the repeatability of flow deficit measurements in normal eyes. They found that the use of a Max projection with a slab positioned 21-31 $\mu \mathrm{m}$ below the RPE band centreline produced the most repeatable results.

The inner choroidal slab demonstrates white and dark regions in which the dark areas represent areas with blood flow below the decorrelation threshold, making the blood flow undetectable on the en face OCTA image. These areas are defined as flow deficits [17-22]. However, one has to consider the possibility that the low signal could be the result of physiologically slow blood flow, and not the result of a specific pathology [17]. Thus to summarize, whether we are actually imaging the precise anatomic CC using OCTA and these various processing methods remains to be confirmed using either adaptive optics indocyanine angiography and/or clinicopathologic correlation. In the interim, the term inner choroidal imaging may be the most appropriate descriptor. However, as the term CC is commonly applied to most OCTA papers on this topic in the literature, we will use the term CC from here on forward in this review in order to maintain consistency with prior publications.

Several studies have shown that these flow deficits may increase with age [23]. The size and extent of the flow deficits may reflect the severity of the pathology or damage to the choriocapillaris, though smaller flow deficits may be physiologic and represent the normal spacing between capillaries - a fact that must be taken into account for quantitative studies of the choriocapillaris [24]. Histologic studies have also revealed that the choriocapillaris vasculature has a varied morphologic appearance in different regions: a dense honeycomb network of freely interconnected capillaries separated by septa in the submacular region versus a polygonal lobular network in the equatorial and peripheral regions $[6,25,26]$.

The intercapillary distance has been noted to also vary by region, ranging 2-20 microns from the center to the periphery [6]. OCTA studies have mirrored these observations and thus it has been proposed to exclude flow deficits smaller than $24 \mu \mathrm{m}$ in diameter as being physiologic when quantifying flow deficits $[22,24,27]$.

A variety of settings and parameters have been used for quantifying choriocapillaris flow deficits (CC FDs) including different axial slab positions, reference offsets, projection artifact removal methods, signal strength, and thresholding strategies [22, 28-34].
Regarding the thresholding strategies, Al Sheikh et al. [19] proposed to use Otsu's global thresholding methodology to detect the CC FDs; however, Otsu's method is based on the assumption of a bimodal distribution for the image histogram which may not be the case for inner choroidal images [35]. Another approach that has been proposed is the use of the mean pixel value in the outer retina layer as a global threshold $[17,36,37]$. Of note, this approach is based on the assumption that the outer retinal layer and inner choroidal layer manifest the same noise level, but that may not be the case due to the presence of the RPE between these two layers. Another quantitative algorithm, the standard deviation (SD) method, is based on the mean and SD from the reference normal database to assess a global threshold. Pixels with an intensity lower than one SD below the mean were considered to represent flow deficits. The main limitation of this method is the lack of a globally approved and validated method to analyze the choriocapillaris [22]. Currently, the most commonly used approach for analysis of the CC/inner choroid is the Phansalkar's local thresholding method $[2,18,20,21,23,34,38-40]$. This approach defines the inner choroidal flow deficits as all areas in which the flow is lower than the given Phansalkar radius set as a threshold. The limitation of this method is that the Phansalkar's radius has to take into consideration the actual pixels size of the image. The final analysis approach is the fuzzy Cmeans self-clustering algorithm that automatically assigns all pixels of the inner choroidal slab into clusters based on the histogram distribution [41].

\section{Choriocapillaris in healthy eyes}

The perfusion of the inner choroid is distinct from other capillary systems as a result of its high flow rate, particularly in the macular region. This high flow rate is necessary in order to provide oxygen and nutrients, and to remove the metabolic waste products. As mentioned previously, the choriocapillaris is historically organized into a series of hexagonal lobules which are smaller in the macular region and become larger toward the periphery (Fig. 1). The intercapillary spaces become wider and progressively elongated toward the periphery [6]. With the progression of high-speed SS-OCTA, a wider investigation of the inner choroid becomes feasible which is essential to understand peripheral chorioretinal disorders. Borrelli et al. reported that the peripapillary region exhibited the greatest total signal void area compared with the macular region and the peripheral region [42]. Within the macular region, the foveal area exhibited a greater total signal void area in comparison with both the parafoveal area and the perifoveal area [42].

In an analysis investigating the near- and mid-peripheral regions, the temporal sector was characterized by a lower total signal void area compared with both the superior and 

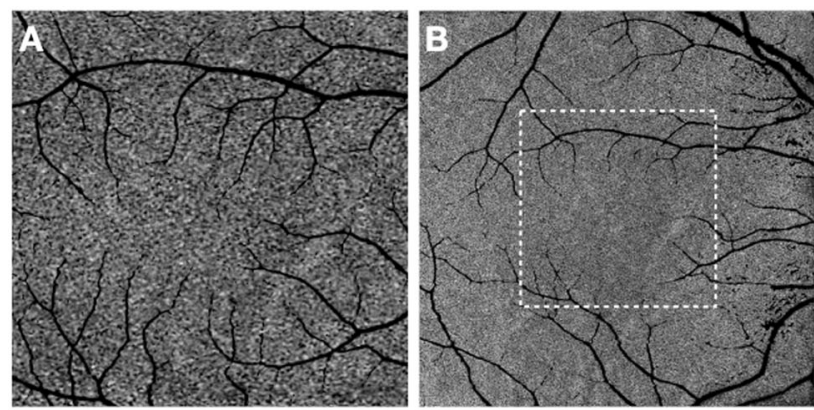

Fig. 1 Choriocapillaris of a healthy eye imaged with swept-source optical coherence tomography angiography (SS OCTA). The $3 \times 3$ (A), $6 \times 6$ (B), $9 \times 9$ (C) and $12 \times 12$-mm (D) OCTA scans show the

inferior areas [42]. Choriocapillaris perfusion seems to have a wide topographic variation in healthy eyes (Fig. 2).

The inner choroidal perfusion in the macular region is inversely correlated with age in both adult and paediatric cohorts, whereas choroidal thickness and gender do not influence the inner choroidal perfusion in healthy subjects [19, 29, 43]. Importantly, while CC FDs do increase with age, the magnitude of the increase is greater centrally $[23,44,45]$.

After this review of the inner choroid in the healthy eye, we now focus on the OCTA findings in the common chorioretinal disorders, including age-related macular degeneration (AMD), diabetic retinopathy (DR), and myopia. Other applications not covered in this review are applications of OCTA imaging of CC in inherited retinal dystrophies and inflammatory disorders.

\section{Choriocapillaris in myopic eyes}

Myopia is a major cause of vision loss worldwide and has increased significantly in prevalence, especially in East and Southeast Asia [46, 47]. Myopia is a risk factor for several macular pathologies, including lacquer crack formation, chorioretinal atrophy, and MNV, which are associated with retinal and choroidal vascular alterations.

Scherm et al. reported that choriocapillary blood flow of the eyes with low myopia $(-1.0 \mathrm{D} \sim-6.0 \mathrm{D})$ did not differ significantly from the emmetropic group and the choriocapillaris seemed to retain a constant level with increasing physiological myopia [48]. However, using an SD-OCTA device, Al Sheikh et al. found an increase in inner choroid flow deficits in highly myopic eyes [49]. Su et al. quantitatively assessed the inner choroid in a cohort of eyes with moderate myopia, high myopia, and age-matched emmetropic normal controls using SS-OCTA [50]. They found the inner choroid in macular regions showed a greater impairment in eyes with high myopia compared to with
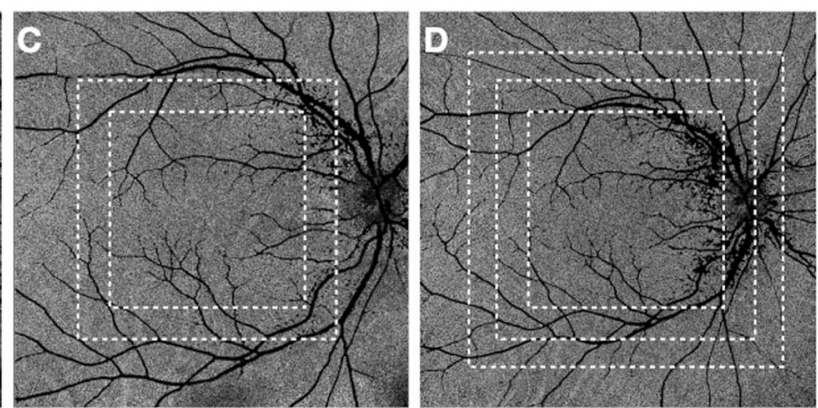

typical granular appearance of the choriocapillaris though the interlacing, anastomotic structure of the choriocapillaris is more difficult to discern with the larger field and consequently lower resolution images.

eyes with lesser degrees of myopia, and these deficits were already present in the absence of features of pathologic or degenerative myopia (Fig. 3) [50]. Interestingly, these studies appear to suggest that changes in the inner choroid are only observed after a certain level of ectasia or progressive myopia is achieved, as individuals with only mild to moderate myopia did not manifest changes to the macular inner choroid. Importantly, more inner choroid flow deficits were observed in these high myopic eyes without any other pathologic alterations (e.g., atrophy, MNV). Assuming inner choroid flow deficits would be worse in eyes with findings of degenerative myopia such as chorioretinal atrophy, one might speculate that progressive CC flow deficit may be a key feature in the evolution and pathophysiology of pathologic myopia. The threshold of inner choroid flow deficits leading to myopic maculopathy remains to be defined.

The alterations of the inner choroid differ depending on the type of myopic maculopathy. OCTA showed complete loss of the choriocapillaris and large choroidal vessels in regions of patchy atrophy with reduced density of the choriocapillaris and reduction in medium and large choroidal vessels in regions of diffuse atrophy [51]. In eyes with lacquer cracks, choriocapillaris rupture appeared to be less advanced compared to the breaks in Bruch's membrane and RPE [51].

Myopic macular neovascularization (MMNV) developing between the RPE and the neurosensory retina (type 2 $\mathrm{MNV}$ ) is a common vision-threatening complication associated with pathologic myopia. Several studies have suggested that OCTA may be a valuable tool for detecting MMNV [52-54]. Two phenotypes of MMNV may be distinguished on OCTA images with different characteristics in size and morphology: the well-organized larger "interlacing" pattern and the small, disorganized CNV "vascular loop" subtype [52]. The limitations of OCTA imaging in highly myopic patients are difficulties in image acquisition and consequent segmentation errors due to the high axial 
Fig. 2 Process for analysing choriocapillaris flow deficits in different macular regions using a multiple concentric rings grid. The superficial capillary plexus (A) is used as reference to center the grid in the center of the foveal avascular zone. The en face

choriocapillaris $(\mathbf{B})$ angiogram is automatically thresholded after masking the superficial vessels in order to obtain a binarized image $(\mathbf{C})$ where the black pixels correspond to the $\mathrm{CC}$ flow deficits (FD). The FDs can be computer within the concentric rings of a grid applied to the image (D). The percentage of flow deficits (FD\%) within each ring may then be plotted as a function of distance from the foveal center defined by radius on the ring.
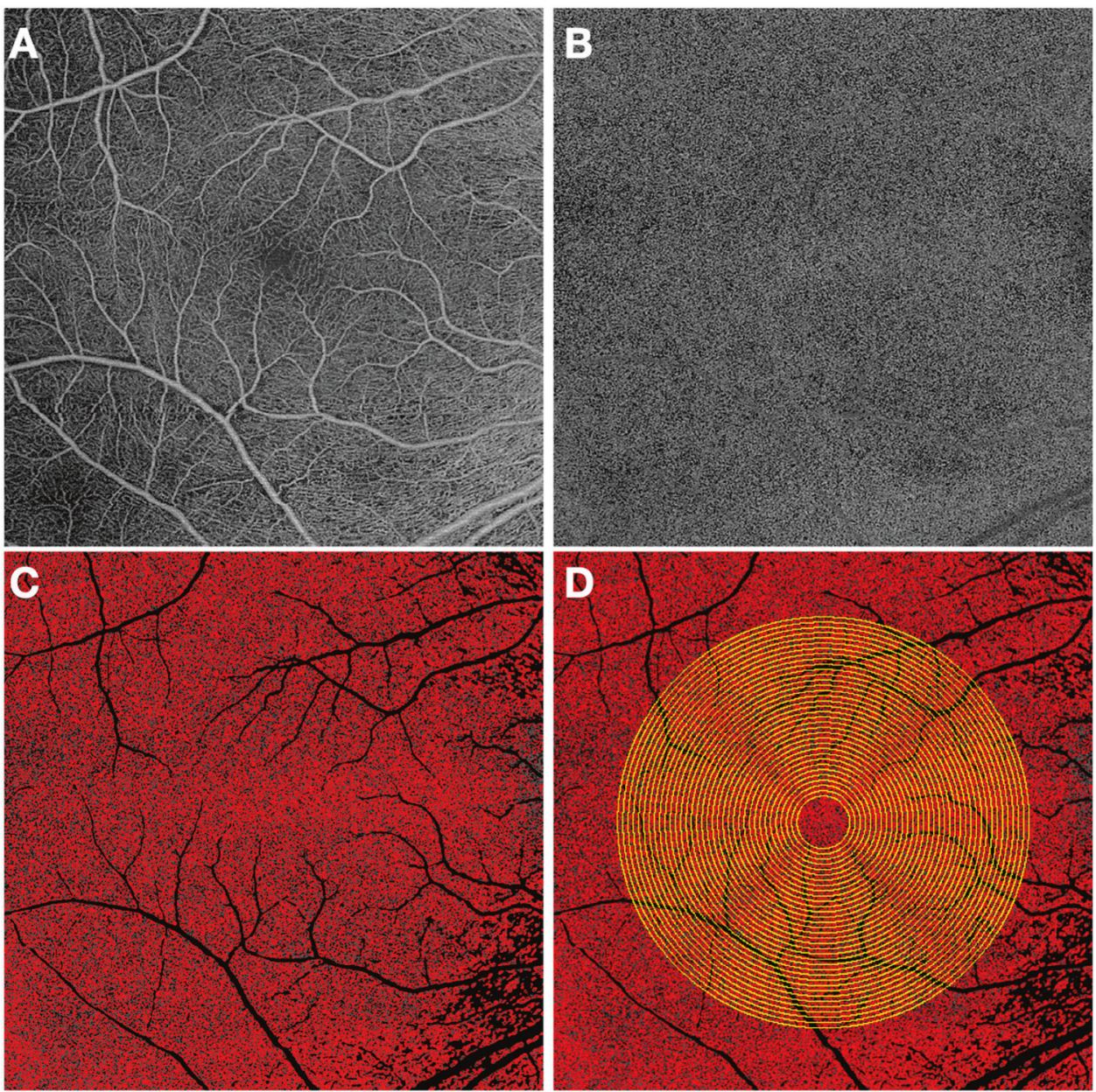

length and fixation errors. To improve image acquisition when a dome-shaped macula and/or posterior staphyloma are present within the region of interest, a manual optimization of focus is helpful. Manual correction of segmentation errors are also frequently needed to accurately visualize the MMNV on OCTA.

\section{Choriocapillaris in DR}

DR, a complication of diabetes mellitus, has become the leading cause of blindness in working-aged people in developed countries. Various studies have shown that both the retinal and choroidal circulations are affected in eyes with DR [2]. OCTA is a useful tool in the detection of retinal vascular changes in DR including clustered capillaries, dilated capillary segments, tortuous capillaries, regions of capillary dropout, reduced capillary density, abnormal capillary loops, foveal avascular zone enlargement, and retinal neovascularization [2, 55-57]. The alteration in the inner choroid has also been illustrated by OCTA. Chio et al. demonstrated that inner choroidal alterations were common not only in non-proliferative diabetic retinopathy (NPDR) and proliferative diabetic retinopathy (PDR), but also in diabetics without retinopathy [58]. In 24 of the 51 eyes from diabetic patients without retinopathy, inner choroidal flow impairment could be detected. This finding is consistent with previous histology, electron microscopy and FA and ICGA studies, suggesting that inner choroidal alterations may occur early in diabetes and play a role in the pathogenesis of DR [59-64].

Nesper et al. found that the inner choroid vascular flow deficit is correlated significantly with disease severity in eyes with DR in a retrospective cross-sectional study including 137 eyes of 86 patients with different stages of DR and 44 eyes of 26 healthy age-matched controls [56]. Another retrospective study showed larger areas of flow voids in the inner choroid in eyes with moderate NPDR, severe NPDR, and PDR compared to those without apparent retinopathy [57]. The non-flow areas in the choriocapillaris layer were associated with poorer VA and disrupted photoreceptor cells, thus indicating their clinical relevance and suggesting the pathological contribution of disrupted choroidal circulation to neuroglial impairment in 
Fig. 3 En face optical coherence tomography angiography images of the choriocapillaris in eyes with myopia. The area of the flow deficit and the size of the flow voids have been shown to be higher in the high myopia eyes (A) compared with moderate myopia (B) and control eyes $(\mathbf{C})$.
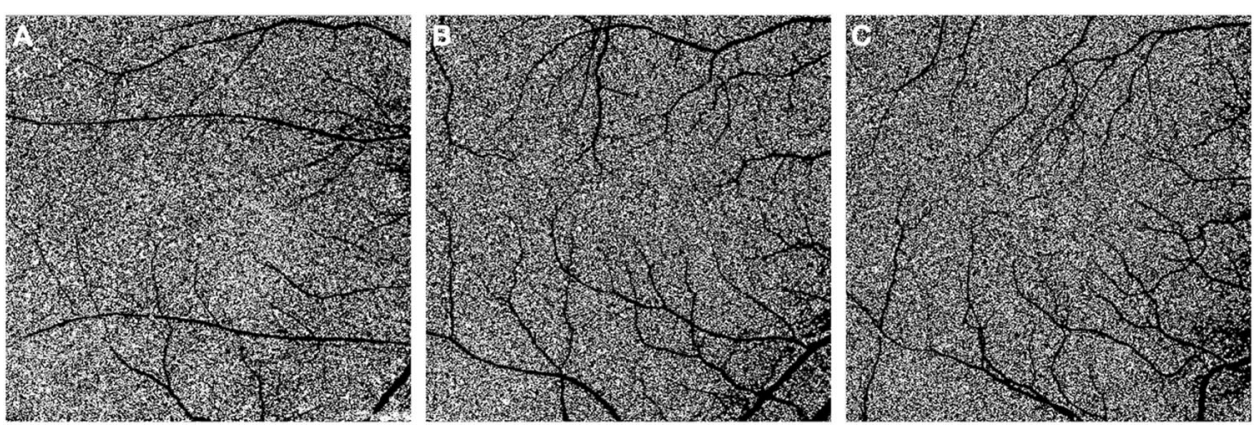

DR [57]. The clinical relevance and pathophysiology of the inner choroidal circulation in eyes with DR merits further longitudinal investigation.

\section{Choriocapillaris in AMD}

AMD is a leading cause of visual impairment among older population in developed contries [65]. Clinically, AMD is classified as early/intermediate AMD (medium and largesized drusen and retinal pigmentary abnormalities) and late AMD (MNV or geographic atrophy (GA)). Severe central vision loss can be the caused by exudation from pathologic type 1 (sub-RPE), type 2 (sub-retinal), or type 3 (intraretinal) $\mathrm{MNV}$ in neovascular AMD or progressive loss of outer retina in GA. Although various factors have been implicated in the pathogenesis of AMD, dysfunction of the photoreceptors, RPE, Bruch's membrane, and choriocapillaris complex is the common pathophysiological abnormality leading to the development of drusen, loss of RPE, and atrophy of photoreceptors.

A critical role for inner choroidal vascular dysfunction in AMD onset and progression has been illustrated by histopathological studies [66, 67]. Seddon et al. demonstrated that extensive inner choroid vascular loss was observed in late-stage GA and MNV patients and the attenuation of the inner choroid progressed with the severity of AMD in early/ intermediate AMD [67]. McLeod et al. investigated the causative relationship between loss of the CC and impairment of the outer retinal layers in a histopathological study [68]. In GA patients, the primary insult appears to be at the level of the RPE and there is an intimate relationship between RPE atrophy and secondary CC degeneration. While in MNV patients, CC degeneration occurs in the presence of viable RPE. The hypoxic RPE resulting from $\mathrm{CC}$ dropout may increase VEGF production and stimulate the outgrowth of MNV.

\section{Choriocapillaris in early/intermediate AMD}

As OCTA technology evolves, inner choroidal measurements may become a useful biomarker for predicting progression of AMD eyes. In a retrospective cohort study using SD OCTA, 42 eyes with intermediate AMD from 42 patients were divided into two subgroups: a group of 20 cases with bilateral intermediate AMD (bilateral intermediate AMD) and 22 cases with neovascular AMD in the fellow eye (unilateral intermediate AMD). A control group of 20 eyes from 20 healthy subjects was included for comparison. In this study, the $\mathrm{CC}$ beneath drusen and under superficial retinal vessels were excluded in order to minimize the impact signal attenuation and projection artifacts (Fig. 4). The average choriocapillaris signal void size was significantly increased in unilateral intermediate AMD eyes compared to both bilateral intermediate AMD and control eyes [69].

Due to the limitation of SD-OCTA systems, most initial studies were limited to an evaluation of the inner choroid in drusen-free regions. With the development of SS-OCTA with a deeper-penetrating wavelength $(1050 \mathrm{~nm})$, reduced sensitivity roll-off, and faster scanning speeds, we can assess the inner choroid even beneath the drusen with more confidence in intermediate AMD eyes. In a cross-sectional study with SS-OCTA, thirty eyes (30 patients) with intermediate AMD and 30 healthy eyes from 30 controls were enrolled [70]. The inner choroid in intermediate AMD eyes demonstrated decreased perfusion with increases in both total area of signal voids and the average signal void area. Furthermore, the impaired CC perfusion was mainly confined to the $\mathrm{CC}$ beneath and surrounding drusen, which was consistent with previous histopathologic observations.

In a retrospective study, Nassisi et al. investigated the inner choroid blood flow in different regions of the macula in eyes with early/intermediate AMD and correlated CC flow deficit in these regions with the subsequent development or enlargement of drusen [71]. Both regions demonstrating new incident drusen or enlargement of existing drusen showed greater inner choroid flow deficits compared to regions which did not show involvement by drusen [71].

\section{Choriocapillaris in neovascular AMD}

One of the most important clinically applications of OCTA is the diagnosis and follow-up of neovascularization in 

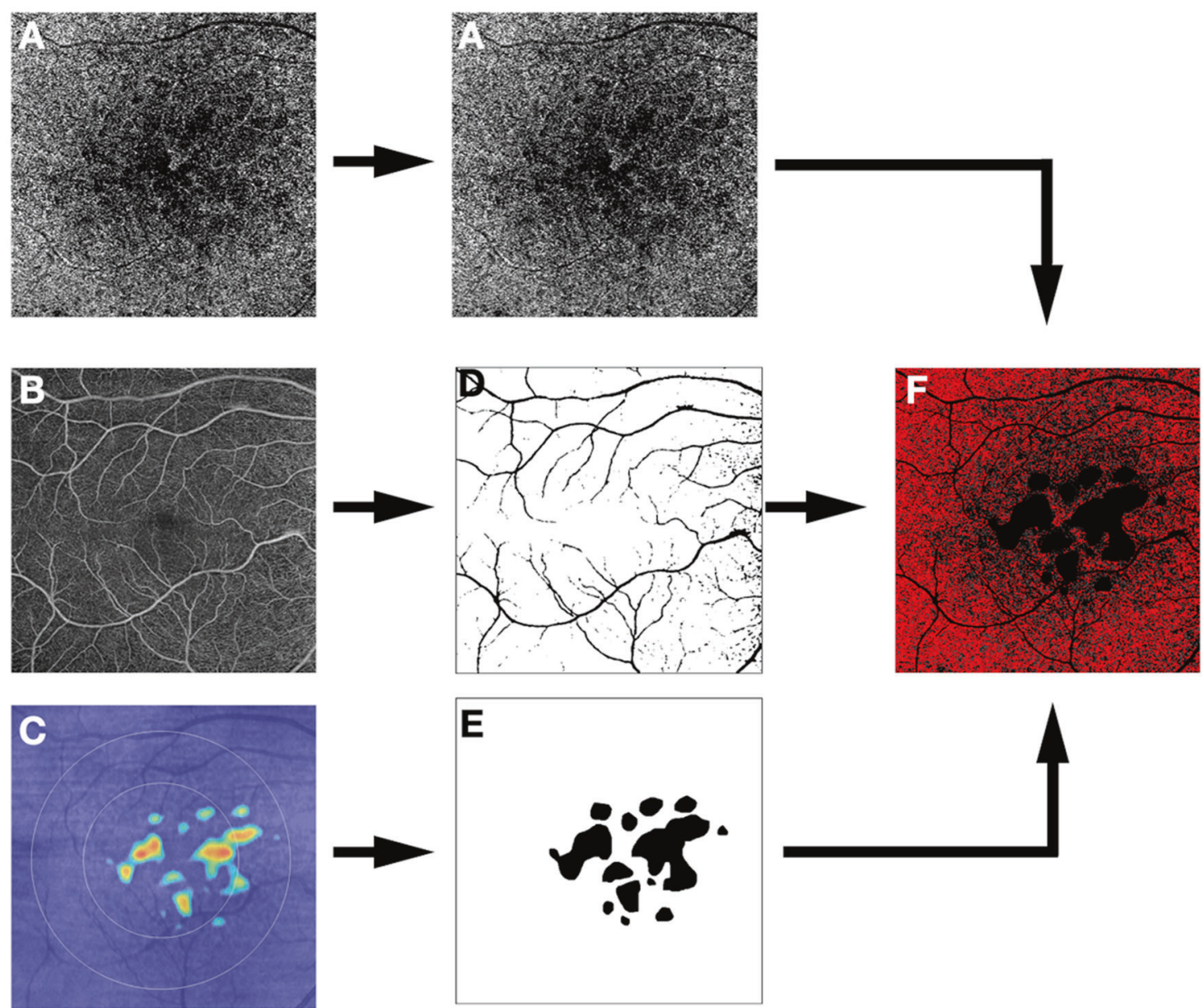

Fig. 4 Strategy for choriocapillaris (CC) analysis in eyes with intermediate AMD. Protocol for spectral domain excluding the drusen and the superficial capillary plexus. En face optical coherence tomography angiography (OCTA) slabs of the choriocapillaris (A) and the superficial capillary plexus (B), as well as the retinal pigment epithelium (RPE) elevation map (C) are exported. The superficial

AMD. The inner choroid in neovascular AMD eyes has also been investigated in several OCTA studies.

A dark ring surrounding type $1 \mathrm{MNV}$ on OCTA has been reported in some studies [72, 73]. The investigators proposed that halo may have been associated with mechanical compression of the underlying inner choroid from the exudative changes leading to alterations in inner choroid blood flow and apparent darkening on OCTA imaging. Another possibility is some type of vascular steal phenomenon due to flow being diverted to the neovascular lesion.

In type $3 \mathrm{MNV}$ eyes, the inner choroid blood flow was investigated and compared with the fellow eyes without MNV [74]. This study demonstrated an overall decreased flow in the inner choroid in eyes with type $3 \mathrm{MNV}$ compared with the fellow eyes without MNV. Importantly, the fellow eyes were compared with the fellow eyes of type 1 or type $2 \mathrm{MNV}$. The total signal void area in the fellow eyes retinal vessels and the RPE elevation map (indicative of regions of drusen or pigment epithelial detachment) are used to create a mask (D, E, respectively) which is applied to the OCTA image to exclude regions which may be confounded by signal attenuation or residual projection artifacts from the analysis $(\mathbf{F})$.

(with type $3 \mathrm{MNV}$ in the other eye) was higher compared with the fellow eyes from unilateral type 1 or type $2 \mathrm{MNV}$ patients. This study indicates the inner choroid blood flow changes in type $3 \mathrm{MNV}$ eyes may be more prominent compared with type 1 or type $2 \mathrm{MNV}$ eyes. The inner choroidal ischemia may induce the production of VEGF and drive the development of type $3 \mathrm{MNV}$. Indeed, the aqueous humor level of VEGF was significantly higher in type $3 \mathrm{MNV}$ eyes compared with eyes with type 1 or type 2 MNV [75].

\section{Choriocapillaris in GA}

GA, characterized by progressive loss of the photoreceptors and RPE, is a significant cause of vision loss in late AMD.

Studies using OCTA have demonstrated severe inner choroid flow impairment within the region of GA [76]. A significant impairment of the inner choroid flow is present 
Fig. 5 En face swept-source optical coherence tomography angiography images of the choriocapillaris in eyes with type 1 macular neovascularization (MNV), geographic atrophy, and normal eye of similar age. The choriocapillaris under the large superficial retinal vessels is excluded from the analysis (D-F). The choriocapillaris surrounding the lesion has been shown to be more severely impaired in the geographic atrophy eyes $(\mathbf{B}, \mathbf{E})$ compared to $\mathrm{MNV}$ eyes $(\mathbf{A}, \mathbf{D})$ or in corresponding regions in normal eyes $(\mathbf{C}, \mathbf{F})$.
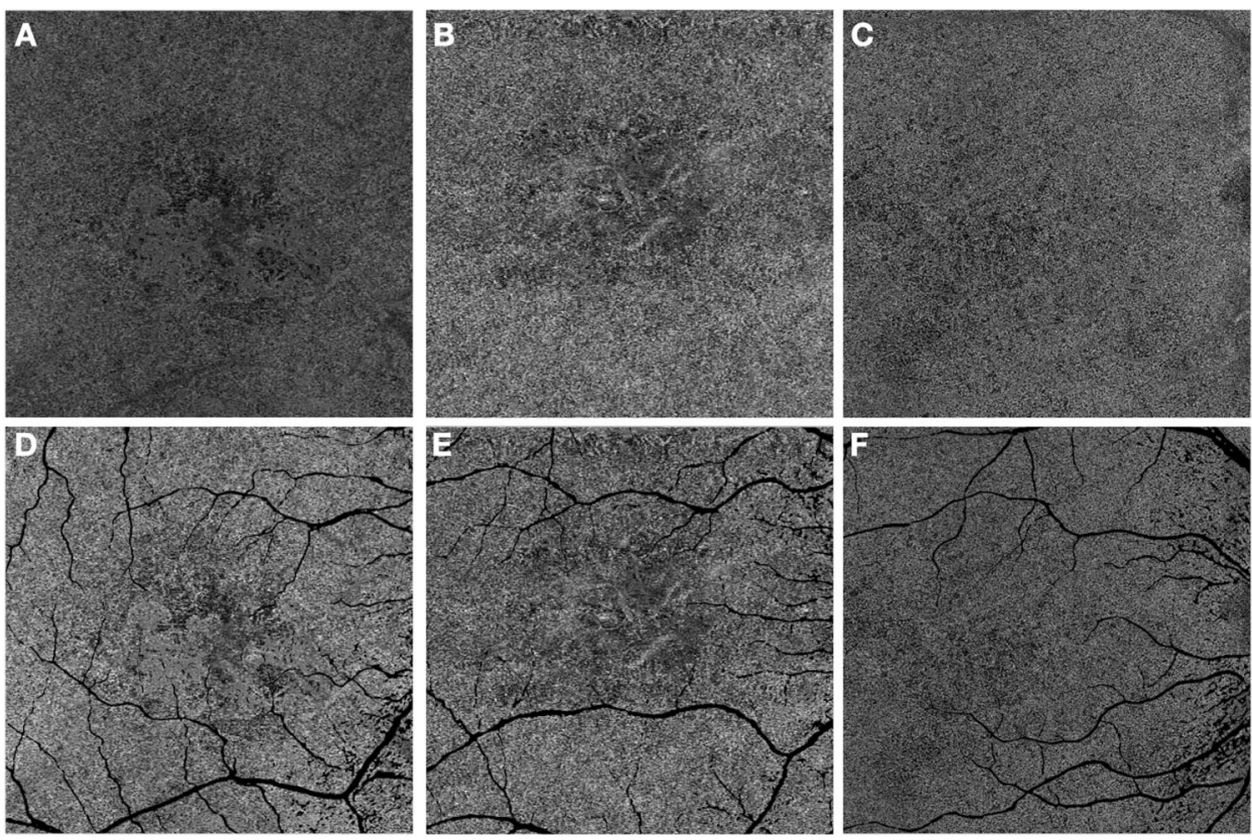

in the zone immediately surrounding the GA lesions [21]. The inner choroid blood flow in peripheral macular regions (peripheral $1 \times 1 \mathrm{~mm}$ squares located at the corners of the $6 \times 6-\mathrm{mm}$ scan images allowing comparison between equidistant regions unaffected by atrophy) in eyes with GA shows greater impairment than in eyes with $\mathrm{CNV}$ and normal eyes [20] (Fig. 5).

The correlation between CC impairment and GA growth rate has become a topic of great interest. In a prospective longitudinal study, 30 eyes of 20 consecutive patients with GA secondary to AMD were included and imaged with SSOCTA [77]. During 1-year follow-up, a greater CC impairment was observed in the area that subsequently developed GA expansion. Nassisi et al. reported a correlation between the CC flow impairment around the atrophic lesions and their yearly growth rate in patients with GA [78]. Alagorie et al. reported only the CC flow deficit in the $500-\mu \mathrm{m}$ region immediately surrounding GA lesions appears to predict the rate of enlargement of these lesions [79].

In contrast to these reports, Thulliez et al. observed the global CC flow deficit in a $6 \times 6 \mathrm{~mm}$ scan had a better correlation with the enlargement rates of GA than those in the regions immediately around the GA [80]. One of the most likely explanation for this outcome is that normal agerelated increases in CC flow deficit within the central macula confound the correlations between the enlargement rates of GA and FDs, whereas the regions furthest away from the margins of GA are less affected by normal agerelated changes and reflect FD alterations related to AMD severity.
Regardless, these studies suggest that the inner choroid flow impairment could predict the enlargement of GA lesion. Thus, the inner choroid impairment could be considered as a new a risk factor for GA progression and a potential biomarker to evaluate the efficacy of novel interventions aiming to slow progression of GA.

\section{Future directions}

Although new insights into the inner choroid in both healthy and diseased eyes have been gained by these early investigations, OCTA is still a fast-developing technology. Artifacts due to segmentation errors, signal attenuation, projection, and eye motion remain challenging $[2,15]$. Recognizing these artifacts is of great importance in avoiding misinterpretation of inner choroidal images. Future progress in OCTA will provide wider field or montage images, more accurate and consistent 3-D projection artifact removal, and artificial intelligence-guided segmentation and image analysis [2]. In summary, an important application of OCTA in the non-invasive imaging of the inner choroid.

\section{Compliance with ethical standards}

Conflict of interest SRS: Consultant: Optos, Centervue, Heidelberg Engineering. Research Instruments: Carl Zeiss Meditec, Nidek, Topcon, Optos, Centervue, Heidelberg Engineering. All other authors: none.

Publisher's note Springer Nature remains neutral with regard to jurisdictional claims in published maps and institutional affiliations. 


\section{References}

1. Bill A, Sperber G, Ujiie K. Physiology of the choroidal vascular bed. Int Ophthalmol. 1983;6:101-7.

2. Spaide RF, Fujimoto JG, Waheed NK, Sadda SR, Staurenghi G. Optical coherence tomography angiography. Prog Retin Eye Res. 2018:64(June 2017):1-55.

3. Nickla DL, Wallman J. The multifunctional choroid. Prog Retin Eye Res. 2010;29:144-68.

4. JW K. The Ocular Circulation. San Rafael (CA): Morgan \& Claypool Life Sciences; 2010.

5. Hayreh SS. Segmental nature of the choroidal vasculature. Br J Ophthalmol. 1975;59:631-48.

6. Olver JM. Functional anatomy of the choroidal circulation: methyl methacrylate casting of human choroid. Eye. 1990;4:262-72.

7. Fryczkowski AW, Sherman MD. Scanning electron microscopy of human ocular vascular casts: the submacular choriocapillaris. Acta Anat. 1988;132:265-9.

8. Krebs W, Krebs IP. Ultrastructural evidence for lymphatic capillaries in the primate choroid. Arch Ophthalmol. 1988;106:1615-6.

9. Spaide RF, Klancnik JMJ, Cooney MJ. Retinal vascular layers imaged by fluorescein angiography and optical coherence tomography angiography. JAMA Ophthalmol. 2015;133:45-50.

10. Matsunaga D, Yi J, Puliafito CA, Kashani AH. OCT angiography in healthy human subjects. Ophthalmic Surg Lasers Imaging Retin. 2014;45:510-5.

11. Moult E, Choi W, Waheed NK, et al. Ultrahigh-speed sweptsource OCT angiography in exudative AMD. Ophthalmic Surg Lasers Imaging Retin. 2014;45:496-505.

12. Corvi F, Pellegrini M, Erba S, Cozzi M, Staurenghi G, Giani A. Reproducibility of vessel density, fractal dimension, and foveal avascular zone using 7 different optical coherence tomography angiography devices. Am J Ophthalmol. 2018;186. https://doi.org/ 10.1016/j.ajo.2017.11.011.

13. Zhang Q, Chen C-L, Chu Z, et al. Automated quantitation of choroidal neovascularization: a comparison study between spectral-domain and swept-source OCT angiograms. Investig Ophthalmol Vis Sci. 2017;58:1506-13.

14. Corvi F, Cozzi M, Barbolini E, et al. Comparison between several optical coherence tomography angiography devices and indocyanine green angiography of choroidal neovascularization. Retina 2020;40:873-80.

15. Spaide RF, Fujimoto JG, Waheed NK. Image artifacts in optical coherence tomography angiography. Retina 2015;35:2163-80.

16. Byon I, Alagorie AR, Ji Y, Su L, Sadda SR. Optimizing the repeatability of choriocapillaris flow deficit measurement from optical coherence tomography angiography. Am J Ophthalmol. 2020. https://doi.org/10.1016/j.ajo.2020.05.027.

17. Borrelli E, Sarraf D, Freund KB, Sadda SR. OCT angiography and evaluation of the choroid and choroidal vascular disorders. Prog Retin Eye Res. 2018;67:30-55.

18. Uji A, Balasubramanian S, Lei J, et al. Multiple enface image averaging for enhanced optical coherence tomography angiography imaging. Acta Ophthalmol. 2018;96:e820-7.

19. Al-Sheikh M, Falavarjani KG, Pfau M, Uji A, Le PP, Sadda SR. Quantitative features of the choriocapillaris in healthy individuals using swept-source optical coherence tomography angiography. Ophthalmic Surg Lasers Imaging Retin. 2017;48: 623-31.

20. Alagorie AR, Verma A, Nassisi M, Sadda SR. Quantitative assessment of choriocapillaris flow deficits in eyes with advanced age-related macular degeneration versus healthy eyes. Am J Ophthalmol. 2019;205:132-9.

21. Nassisi M, Shi Y, Fan W, et al. Choriocapillaris impairment around the atrophic lesions in patients with geographic atrophy: a swept-source optical coherence tomography angiography study. Br J Ophthalmol. 2019;103:911-7.

22. Zhang Q, Zheng F, Motulsky EH, et al. A Novel strategy for quantifying choriocapillaris flow voids using swept-source OCT angiography. Investig Ophthalmol Vis Sci. 2018;59:203-11.

23. Nassisi M, Baghdasaryan E, Tepelus T, Asanad S, Borrelli E, Sadda SR. Topographic distribution of choriocapillaris flow deficits in healthy eyes. PLoS ONE. 2018;13:e0207638.

24. Zhang Q, Shi Y, Zhou H, et al. Accurate estimation of choriocapillaris flow deficits beyond normal intercapillary spacing with swept source OCT angiography. Quant Imaging Med Surg. 2018;8:658-66.

25. Torczynski E, Tso MO. The architecture of the choriocapillaris at the posterior pole. Am J Ophthalmol. 1976;81:428-40.

26. Yoneya S, Tso MO, Shimizu K. Patterns of the choriocapillaris. A method to study the choroidal vasculature of the enucleated human eye. Int Ophthalmol. 1983;6:95-9.

27. Choi W, Mohler KJ, Potsaid B, et al. Choriocapillaris and choroidal microvasculature imaging with ultrahigh speed OCT angiography. PLoS ONE. 2013;8:e81499.

28. Bhutto I, Lutty G. Understanding age-related macular degeneration (AMD): relationships between the photoreceptor/retinal pigment epithelium/Bruch's membrane/choriocapillaris complex. Mol Asp Med. 2012;33:295-317.

29. Spaide RF. Choriocapillaris flow features follow a power law distribution: implications for characterization and mechanisms of disease progression. Am J Ophthalmol. 2016;170:58-67.

30. Uji A, Balasubramanian S, Lei J, Baghdasaryan E, Al-Sheikh M, Sadda SR. Impact of multiple En face image averaging on quantitative assessment from optical coherence tomography angiography images. Ophthalmology. 2017;124:944-52.

31. Uji A, Balasubramanian S, Lei J, Baghdasaryan E, Al-Sheikh M, Sadda SR. Choriocapillaris imaging using multiple En face optical coherence tomography angiography image averaging. JAMA Ophthalmol. 2017;135:1197-204.

32. Byon I, Nassisi M, Borrelli E, Sadda SR. Impact of slab selection on quantification of choriocapillaris flow deficits by optical coherence tomography angiography. Am J Ophthalmol. 2019;208:397-405.

33. Rochepeau C, Kodjikian L, Garcia M-A, et al. Optical coherence tomography angiography quantitative assessment of choriocapillaris blood flow in central serous chorioretinopathy. Am J Ophthalmol. 2018;194:26-34.

34. Zheng F, Zhang Q, Shi Y, et al. Age-dependent changes in the macular choriocapillaris of normal eyes imaged with swept-source optical coherence tomography angiography. Am J Ophthalmol. 2019;200:110-22.

35. N. O. A threshold selection method from gray-level histograms. IEEE Trans Syst Man Cyber. 1979;9:62-6.

36. Nesper PL, Soetikno BT, Fawzi AA. Choriocapillaris nonperfusion is associated with poor visual acuity in eyes with reticular pseudodrusen. Am J Ophthalmol. 2017;174:42-55.

37. Alten F, Heiduschka P, Clemens CR, Eter N. Exploring choriocapillaris under reticular pseudodrusen using OCT-angiography. Graefe's Arch Clin Exp Ophthalmol. 2016;254:2165-73.

38. Neerad Phansalkar N, Sumit More S, Ashish Sabale A, Madhuri Joshi M. Adaptive local thresholding for detection of nuclei in diversity stained cytology images. In: 2011 International conference on communications and signal processing. IEEE; 2011. p. 218-20. https://doi.org/10.1109/ICCSP.2011.5739305.

39. Spaide RF. Choriocapillaris signal voids in maternally inherited diabetes and deafness and in pseudoxanthoma elasticum. Retina. 2017;37:2008-14

40. Chu Z, Gregori G, Rosenfeld PJ, Wang RK. Quantification of choriocapillaris with optical coherence tomography angiography: a comparison study. Am J Ophthalmol. 2019;208:111-23. 
41. Chu Z, Zhou H, Cheng Y, Zhang Q, Wang RK. Improving visualization and quantitative assessment of choriocapillaris with swept source OCTA through registration and averaging applicable to clinical systems. Sci Rep. 2018;8:16826.

42. Borrelli E, Uji A, Toto L, Viggiano P, Evangelista F, Mastropasqua $\mathrm{R}$. In vivo mapping of the choriocapillaris in healthy eyes: a widefield swept-source OCT angiography study. Ophthalmol Retin. 2019;3:979-84.

43. Wang Q, Chan S, Yang JY, et al. Vascular density in retina and choriocapillaris as measured by optical coherence tomography angiography. Am J Ophthalmol. 2016;168:95-109.

44. Shi Y, Zhang Q, Zheng F, et al. Correlations between different choriocapillaris flow deficit parameters in normal eyes using swept source OCT angiography. Am J Ophthalmol. 2020;209:18-26.

45. Zhou H, Dai Y, Shi Y, et al. Age-related changes in choroidal thickness and the volume of vessels and stroma using sweptsource OCT and fully automated algorithms. Ophthalmol Retin. 2020;4:204-15.

46. Fricke TR, Jong M, Naidoo KS, et al. Global prevalence of visual impairment associated with myopic macular degeneration and temporal trends from 2000 through 2050: systematic review, meta-analysis and modelling. Br J Ophthalmol. 2018;102:855-62.

47. Morgan IG, French AN, Ashby RS, et al. The epidemics of myopia: aetiology and prevention. Prog Retin Eye Res. 2018;62:134-49.

48. Scherm P, Pettenkofer M, Maier M, Lohmann CP, Feucht N. Choriocapillary blood flow in myopic subjects measured with OCT angiography. Ophthalmic Surg Lasers Imaging Retin. 2019;50:e133-9.

49. Al-Sheikh M, Phasukkijwatana N, Dolz-Marco R, et al. Quantitative OCT angiography of the retinal microvasculature and the choriocapillaris in myopic eyes. Invest Ophthalmol Vis Sci. 2017;58:2063-9.

50. Su L, Ji Y-S, Tong N, et al. Quantitative assessment of the retinal microvasculature and choriocapillaris in myopic patients using swept-source optical coherence tomography angiography. Graefe's Arch Clin Exp Ophthalmoll. 2020;258:1173-80.

51. Sayanagi K, Ikuno Y, Uematsu S, Nishida K. Features of the choriocapillaris in myopic maculopathy identified by optical coherence tomography angiography. $\mathrm{Br} \quad \mathrm{J}$ Ophthalmol. 2017;101:1524-9.

52. Bruyère E, Miere A, Cohen SY, et al. Neovascularization secondary to high myopia imaged by optical coherence tomography angiography. Retina. 2017;37:2095-101.

53. Querques L, Giuffrè C, Corvi F, et al. Optical coherence tomography angiography of myopic choroidal neovascularisation. $\mathrm{Br} \mathrm{J}$ Ophthalmol. 2017;101. https://doi.org/10.1136/bjophthalmol2016-309162.

54. Querques G, Corvi F, Querques L, Souied EH, Bandello F. Optical coherence tomography angiography of choroidal neovascularization secondary to pathologic myopia. 2016;56. https:// doi.org/10.1159/000442800.

55. Parrulli S, Corvi F, Cozzi M, Monteduro D, Zicarelli F, Staurenghi G. Microaneurysms visualisation using five different optical coherence tomography angiography devices compared to fluorescein angiography. Br J Ophthalmol. 2020. https://doi.org/10. 1136/bjophthalmol-2020-316817.

56. Nesper PL, Roberts PK, Onishi AC, et al. Quantifying microvascular abnormalities with increasing severity of diabetic retinopathy using optical coherence tomography angiography. Investig Ophthalmol Vis Sci. 2017;58:BIO307-15.

57. Dodo Y, Suzuma K, Ishihara K, et al. Clinical relevance of reduced decorrelation signals in the diabetic inner choroid on optical coherence tomography angiography. Sci Rep. 2017;7:5227.
58. Choi W, Waheed NK, Moult EM, et al. Ultrahigh speed swept source optical coherence tomography angiography of retinal and choriocapillaris alterations in diabetic patients with and without retinopathy. Retina. 2017;37:11-21.

59. Fryczkowski AW, Hodes BL, Walker J. Diabetic choroidal and iris vasculature scanning electron microscopy findings. Int Ophthalmol. 1989;13:269-79.

60. Lutty GA, Cao J, McLeod DS. Relationship of polymorphonuclear leukocytes to capillary dropout in the human diabetic choroid. Am J Pathol. 1997;151:707-14.

61. Cao J, McLeod S, Merges CA, Lutty GA. Choriocapillaris degeneration and related pathologic changes in human diabetic eyes. Arch Ophthalmol. 1998;116:589-97.

62. Gerl VB, Bohl J, Pitz S, Stoffelns B, Pfeiffer N, Bhakdi S. Extensive deposits of complement C3d and C5b-9 in the choriocapillaris of eyes of patients with diabetic retinopathy. Investig Ophthalmol Vis Sci. 2002;43:1104-8.

63. Hua R, Liu L, Wang X, Chen L. Imaging evidence of diabetic choroidopathy in vivo: angiographic pathoanatomy and choroidalenhanced depth imaging. PLoS ONE. 2013;8:e83494.

64. Shiragami C, Shiraga F, Matsuo T, Tsuchida Y, Ohtsuki H. Risk factors for diabetic choroidopathy in patients with diabetic retinopathy. Graefe's Arch Clin Exp Ophthalmol. 2002;240:436-42.

65. Mitchell P, Liew G, Gopinath B, Wong TY. Age-related macular degeneration. Lancet. 2018;392:1147-59.

66. Mullins RF, Johnson MN, Faidley EA, Skeie JM, Huang J. Choriocapillaris vascular dropout related to density of drusen in human eyes with early age-related macular degeneration. Investig Ophthalmol Vis Sci. 2011;52:1606-12.

67. Seddon JM, McLeod DS, Bhutto IA, et al. Histopathological insights into choroidal vascular loss in clinically documented cases of age-related macular degeneration. JAMA Ophthalmol. 2016;134:1272-80.

68. McLeod DS, Grebe R, Bhutto I, Merges C, Baba T, Lutty GA. Relationship between RPE and choriocapillaris in age-related macular degeneration. Investig Ophthalmol Vis Sci. 2009;50:4982-91.

69. Borrelli E, Uji A, Sarraf D, Sadda SR. Alterations in the choriocapillaris in intermediate age-related macular degeneration. Investig Ophthalmol Vis Sci. 2017;58:4792-8.

70. Borrelli E, Shi Y, Uji A, et al. Topographic analysis of the choriocapillaris in intermediate age-related macular degeneration. Am J Ophthalmol. 2018;196:34-43.

71. Nassisi M, Tepelus T, Nittala MG, Sadda SR. Choriocapillaris flow impairment predicts the development and enlargement of drusen. Graefe's Arch Clin Exp Ophthalmol. 2019;257:2079-85.

72. Inoue $\mathrm{M}$, Jung JJ, Balaratnasingam $\mathrm{C}$, et al. A comparison between optical coherence tomography angiography and fluorescein angiography for the imaging of type 1 neovascularization. Investig Ophthalmol Vis Sci. 2016;57:OCT314-23.

73. Jia Y, Bailey ST, Wilson DJ, et al. Quantitative optical coherence tomography angiography of choroidal neovascularization in agerelated macular degeneration. Ophthalmology. 2014;121:1435-44.

74. Borrelli E, Souied EH, Freund KB, et al. Reduced choriocapillaris flow in eyes with type 3 neovascularization and age-related macular degeneration. Retina. 2018;38:1968-76.

75. dell'Omo R, Cassetta M, dell'Omo E, et al. Aqueous humor levels of vascular endothelial growth factor before and after intravitreal bevacizumab in type 3 versus type 1 and 2 neovascularization. A prospective, case-control study. Am J Ophthalmol. 2012;153:155-61.e2.

76. Choi W, Moult EM, Waheed NK, et al. Ultrahigh-speed, sweptsource optical coherence tomography angiography in nonexudative age-related macular degeneration with geographic atrophy. Ophthalmology. 2015;122:2532-44. 
77. Sacconi R, Corbelli E, Borrelli E, et al. Choriocapillaris flow impairment could predict the enlargement of geographic atrophy lesion. Br J Ophthalmol. 2020. https://doi.org/10.1136/bjophtha 1mol-2019-315800.

78. Nassisi M, Baghdasaryan E, Borrelli E, Ip M, Sadda SR. Choriocapillaris flow impairment surrounding geographic atrophy correlates with disease progression. PLOS ONE. 2019;14: e0212563.
79. Alagorie AR, Nassisi M, Verma A, et al. Relationship between proximity of choriocapillaris flow deficits and enlargement rate of geographic atrophy. Graefe's Arch Clin Exp Ophthalmol. 2020;258:995-1003.

80. Thulliez M, Zhang Q, Shi Y, et al. Correlations between choriocapillaris flow deficits around geographic atrophy and enlargement rates based on swept-source OCT imaging. Ophthalmol Retin. 2019;3:478-88. 